\title{
Role and Mechanism of LIF in Oral Squamous Cell Carcinoma Progression
}

\author{
Ting-An Lin ${ }^{1}$, Tai-Sheng $\mathrm{Wu}^{2}$, Yue-Ju $\mathrm{Li}^{2,3}$, Cheng-Ning Yang ${ }^{2}$, Monica Maria Illescas Ralda ${ }^{2}$ \\ and Hao-Hueng Chang 2,4,* \\ 1 Graduate Institute of Oral Biology, School of Dentistry, National Taiwan University, Taipei 100, Taiwan; \\ ting4357025@gmail.com \\ 2 Graduate Institute of Clinical Dentistry, School of Dentistry, National Taiwan University, Taipei 100, Taiwan; \\ eric12345a@hotmail.com (T.-S.W.); anaballlee@yahoo.com.tw (Y.-J.L.); cnyang880@yahoo.com.tw (C.-N.Y.); \\ mmillescas@ufm.edu (M.M.I.R.) \\ 3 Department of Surgery, National Taiwan University Hospital, Taipei 100, Taiwan \\ 4 Department of Dentistry, National Taiwan University Hospital, Taipei 100, Taiwan \\ * Correspondence: changhh@ntu.edu.tw; Tel.: +886-2-23123456-66847; Fax: +886-2-23831346
}

Received: 6 January 2020; Accepted: 19 January 2020; Published: 21 January 2020

\begin{abstract}
Background: Metastasis is a severe problem in patients with oral squamous cell carcinoma (OSCC), which is the fifth most common cancer worldwide. Leukemia inhibitory factor (LIF) has been studied in different cancers, while the role of LIF in OSCC remains unclear. Methods: LIF expression was detected in 100 OSCC samples by immunohistochemistry. Effects of LIF on cell motility were evaluated in OSCC cell lines. High-throughput microarray analysis was also conducted. The correlation between LIF and the downstream effector was analyzed by real-time quantitative reverse transcription PCR. Results: Patients with OSCC who had lymph node metastasis or advanced cancer stages showed high LIF expression. OSCC patients with higher LIF expression, advanced stage, large tumor size, or lymph node metastasis had significantly shorter overall survival. LIF regulated cancer cell motilities through outside-in signaling. The inhibin beta A subunit (INHBA) gene was identified as a crucial downstream effector of LIF-promoted OSCC progression and restored migration and invasion abilities in LIF knockdown transfectants. Conclusion: LIF enhances regional lymphatic spread, thus leading to an advanced cancer stage. Regulation of LIF downstream molecules such as INHBA inhibits the invasion or migration ability of cancer cells. Thus, LIF can be a potential target in preventing cancer metastasis and spread.
\end{abstract}

Keywords: oral cancer; LIF; INHBA; migration; invasion

\section{Introduction}

Oral cancer is the sixth leading cause of malignant tumor mortality and is an important healthcare problem worldwide [1]. Oral squamous cell carcinoma (OSCC) accounts for more than $90 \%$ of all oral cancers, with over 300,000 new cases being registered annually [2,3]. Oral cancer is an umbrella term for cancers that originate in sites such as the tongue, lip, gums, buccal mucosa, floor of the mouth, oropharynx, gingiva, and hard palate [2,4]. The risk factors for oral malignancy are tobacco smoking, alcoholism, an unhealthy diet, and viral infections [2,4]. Unlike in Western countries, in Southeast Asia and Taiwan, extensive epidemiologic evidence has demonstrated a close relationship between the development of OSCC and areca nut chewing, tobacco smoking, and alcohol consumption [5-7]. According to a report by the Ministry of Health and Welfare, Taiwan, oral cancer has the seventh highest incidence and the sixth highest mortality rate of all cancers [8,9].

In Taiwan, those who chew betel nuts have a higher relative risk of oral cancer than those who do not. Arecoline, the main component of areca nut extract, is a major risk factor for oral 
premalignant lesions and considerably increases OSCC-related carcinogenesis [10]. In addition, areca nut components stimulate epidermal growth factor receptor (EGFR) phosphorylation and interleukin (IL)-1 $\alpha$ production and induce reactive oxygen species in oral submucous fibrosis [11]. Tobacco smoking and alcohol consumption are also key risk factors for oral cancer in Taiwan. Smokers have a higher risk of potentially malignant oral disorders (hazard ratio of 2.8) than nonsmokers [12]. ADH1B, one of the genes encoding the ADH enzyme, and $A L D H 2$, a gene that encodes an enzyme responsible for the conversion of acetaldehyde to acetate, may be associated with head and neck cancer in alcohol drinkers [13].

Leukemia inhibitory factor (LIF) is a pleiotropic proinflammatory cytokine in the IL-6 group. LIF is widely expressed in different types of tissues and regulates multiple vital biological functions such as survival, differentiation, and inflammatory response [14,15]. It is so named because of its function of inducing the differentiation of murine M1 myeloid leukemia cells and macrophage maturation to suppress leukemia proliferation [16]. LIF binds to the LIF receptor (LIFR) and glycoprotein (gp130, IL6ST) to form a high-affinity receptor complex. This heterodimer then activates many downstream signaling pathways of LIF, including JAK/STAT3, PI3K/AKT, MAPK/ERK, and mTOR, and regulates transcription [17-20].

LIF has been reported to have distinct effects on tumorigenesis and metastatic spread in different cancers. It exerts tumor-suppressing effects through TGF- $\beta$-induced cell cycle arrest and inhibition of cell migration through the LIF/p21 signaling cascade pathway in cutaneous melanoma [21]. Chen et al. found that LIFR suppresses breast cancer metastasis by triggering the Hippo-YAP pathway to inhibit local invasion and metastatic colonization [22,23]. LIF is also considered to be an oncogene that promotes the development and progression of many types of solid tumors. LIF and LIFR are overexpressed in nasopharyngeal carcinoma and were shown to enhance radioresistance through activating mTOR and p70S6K [18]. In rhabdomyosarcoma, LIF stimulates AKT phosphorylation and strongly enhances the invasive potential of tumors [24]. Furthermore, LIF overexpression can promote colorectal cancer chemoresistance in a p53-dependent manner [25].

Although LIF has been indicated as a novel biomarker and a prognostic target in various human cancers, its biological functions in oral cancer remain unknown. Also, the relationship between LIF and the risk factors of oral cancer such as tobacco smoking, betel nut chewing, and alcohol consumption is unclear. In this study, we evaluated the correlation between LIF expression and the clinicopathological parameters of patients with OSCC and investigated whether LIF drives tumor progression in oral cancer.

\section{Materials and Methods}

\subsection{Cell Lines and Culture}

SAS (JCRB 0260), CA9-22 (JCRB 0625), and Cal27 (ATCC CRL-2095) purchased from ATCC were used in this study. All cell lines were cultured in Dulbecco's modified Eagle's medium (Gibco, Grand Island, NY, USA) supplemented with 10\% fetal bovine serum (Gibco) and 1\% antibiotics (Gibco) at $37{ }^{\circ} \mathrm{C}$ in a humidified atmosphere of $5 \% \mathrm{CO}_{2}$ and $95 \%$ air. For routine culture and harvesting, adherent cells were trypsinized from culture dishes with $0.05 \%$ trypsin/EDTA (Gibco).

\subsection{Transient Transfection and Stable Transfection Clone Selection}

shLIF plasmids (Academia Sinica RNAi Core Lab, Taipei, Taiwan), LIF (Origene, Rockville, MD, USA, NM_002309), and inhibin beta A subunit (INHBA) (Origene, NM_002192) expression plasmids were transiently transfected into OSCC cell lines by Lipofectamine 2000 (Invitrogen, Carlsbad, CA, USA), and the transfection protocol was conducted according to the manufacturer's instructions (Invitrogen, Carlsbad, CA, USA). Integration of transfectant plasmid DNA was confirmed by Western blotting. After transection for $24 \mathrm{~h}$, the stable transfectant was selected in puromycin at a concentration of $3 \mu \mathrm{g} / \mathrm{mL}$. 


\subsection{Western Blotting}

Cells were collected with a lysis buffer. Proteins $(50 \mu \mathrm{g})$ were separated by sodium-dodecylsulfate-polyacrylamide-gel-electrophoresis on $10 \%$ gels and electrotransferred to a polyvinylidene difluoride (PVDF) membrane (Millipore, Bedford, MA, USA). After the blot was blocked in $5 \%$ skim milk with $0.1 \%$ Tween-20, membrane-bound proteins were probed with primary antibodies at $4{ }^{\circ} \mathrm{C}$ overnight and incubated with peroxidase-conjugated polyclonal secondary antibodies (1:3000) for $1 \mathrm{~h}$ at room temperature. Antibody-bound protein bands were detected with enhanced chemiluminescence reagents (Millipore) and photographed with an LAS-4000 (Fujifilm, Tokyo, Japan). LIF (Abcam, Cambridge, UK, ab135629), INHBA (R \& D systems, Minneapolis, MN USA, AF338), and $\beta$-actin (Santa Cruz Biotechnology, Dallas, TX, USA, sc-47778) antibodies were used for the Western blotting analysis.

\subsection{Immunohistochemistry}

Immunohistochemical studies were conducted using the ss-polymer HRP detection kit (Biogenex, San Ramon, CA, USA). Briefly, after being deparaffinized and rehydrated, the sections were subjected to $10 \mathrm{~min}$ of heat-induced antigen retrieval in citrate buffer $(\mathrm{pH}$ 6.0). After being blocked by $3 \%$ hydrogen peroxide, the sections (slides) were incubated with primary antibodies (monoclonal anti-LIF (1:100)) at $4{ }^{\circ} \mathrm{C}$ overnight. Then, the slides were incubated for $60 \mathrm{~min}$ with super enhancer and $20 \mathrm{~min}$ with the polymer HRP. Antigen-antibody complexes were visualized with 3.3 hematoxylin, differentiated, dehydrated, and mounted. A routinely processed OSCC that was previously proven to be positive for LIF was used as a positive control, and the OSCC section without a primary antibody served as a negative control in each staining series. The grade of LIF protein expression in each specimen was further classified by intensity level, namely, 0 (negative or $<5 \%$ of tumor cells stained), 1 (5-25\% of tumor cells stained), 2 ( $26-50 \%$ of tumor cells stained), 3 (51-75\% of tumor cells stained), and 4 ( $>76 \%$ of tumor cells stained). Two authors (H.-H.C. and M.M.I.R.) reviewed the LIF staining on each slide independently without knowledge of the patients' information.

\subsection{In Vitro Migration and Invasion Assay}

For in vitro cell invasion and migration assays, Transwell chambers $(8 \mu \mathrm{m}$ pore size; Corning Costar, Cambridge, MA, USA) coated with or without Matrigel (Corning, New York, NY, USA) in 24 -well dishes were used. Cells were allowed to grow to subconfluency $(\sim 80-90 \%)$. After detachment with trypsin, cells resuspended in a serum-free medium and $100 \mu \mathrm{L}$ cell suspension $\left(1 \times 10^{5}\right.$ cells $\left./ \mathrm{mL}\right)$ were added to the upper chamber. A complete medium was added to the bottom wells of the chambers. After $24 \mathrm{~h}$, the cells were fixed in ice methanol for $15 \mathrm{~min}$ and the upper side cells of the filters were removed with cotton-tipped swabs. The filters were then cleared with phosphate-buffered saline (PBS) and the cells were stained with $0.05 \%$ crystal violet in PBS for $20 \mathrm{~min}$. The underside cells of the filters were viewed, images of 10 different fields were captured from each membrane, and the number of migratory cells was counted. The mean of triplicate assays for each experimental condition was used.

\subsection{RNA Isolation}

RNA was isolated from oral carcinoma cells and samples from patients with OSCC by TRIzol (Invitrogen, Carlsbad, CA, USA). Reverse transcription was performed in a final reaction containing the following: total RNA ( $5 \mu \mathrm{g})$, First Strand Buffer with DTT (10 mM), deoxyribonucleotide triphosphate (dNTP; $2.5 \mathrm{mM})$, Oligo $(\mathrm{dT})$ 12-18 primer $(1 \mu \mathrm{g})$, and Moloney murine leukemia virus reverse transcriptase $(200 \mathrm{U})$. The reaction was incubated at $65{ }^{\circ} \mathrm{C}$ for $5 \mathrm{~min}$ and then was terminated by heating at $42{ }^{\circ} \mathrm{C}$ for $1 \mathrm{~h}$.

\subsection{Reverse Transcription PCR}

PCR amplification was conducted in a reaction buffer containing $20 \mathrm{mM}$ of Tris- $\mathrm{HCl}$ (pH 8.4), $50 \mathrm{mM}$ of $\mathrm{KCl}, 1.5 \mathrm{mM}$ of $\mathrm{MgCl}_{2}, 167 \mu \mathrm{M}$ of dNTPs, $2.5 \mathrm{U}$ of Taq DNA polymerase, and $0.1 \mu \mathrm{M}$ of 
primers. The reactions were performed in a Biometra Thermoblock (Biometra Inc., Baltimore, MD, USA) using the following process: denaturing for $1 \mathrm{~min}$ at $95^{\circ} \mathrm{C}$, annealing for $1 \mathrm{~min}$ at $58{ }^{\circ} \mathrm{C}$, and elongating for $1 \mathrm{~min}$ at $72{ }^{\circ} \mathrm{C}$ ( 30 cycles in total); the final extension occurred at $72{ }^{\circ} \mathrm{C}$ for $10 \mathrm{~min}$. Equal volumes of each sample were subjected to electrophoresis on a $1 \%$ agarose gel, which was then stained with ethidium bromide and photographed under UV illumination.

\subsection{Real-Time Quantitative Reverse Transcription PCR}

The complementary DNA was used as a template in real-time quantitative PCR reactions with LIF, INHBA, and GAPDH primers using an ECO Sequence detector (Illumina, San Diego, CA, USA) at $95^{\circ} \mathrm{C}$ for $10 \mathrm{~min}$, followed by 40 cycles of $95^{\circ} \mathrm{C}$ for $15 \mathrm{~s}$ and $60^{\circ} \mathrm{C}$ for $1 \mathrm{~min}$. Target gene expression was normalized between different samples based on the values of GAPDH RNA expression.

\subsection{OSCC Tumor Samples and Clinical Data Collection}

OSCC specimens were collected at the time of surgery from previously untreated patients who underwent surgical resection at National Taiwan University Hospital (201503035RINC). The trial was approved by the Institutional Review Board of National Taiwan University Hospital (registration 201503035RINC). All participants provided informed consent before participating in the trial. We obtained formalin-fixed, paraffin-embedded specimens from 100 patients ( 91 men and 9 women, mean age of 55.8 years, range of 34-82 years) with OSCC. The diagnosis of OSCC was based on the histological examination of hematoxylin-and-eosin-stained tissue sections. All patients underwent total surgical excision of their OSCCs at the Department of Oral and Maxillofacial Surgery of National Taiwan University Hospital, Taipei, Taiwan. None of the patients had received any form of tumor-specific therapy prior to total surgical excision of their lesions. Specimens were obtained from the total surgical excision of the lesions. Of the 100 cases of OSCC, $48(48 \%)$ were located in the buccal mucosa, 34 (34\%) on the tongue, $12(12 \%)$ on the gingiva, $5(5 \%)$ on the hard palate, and $1(1 \%)$ on the floor of the mouth. All of the specimens were snap-frozen immediately and stored at $-80^{\circ} \mathrm{C}$. The histologic identification of oral cancer was determined as recommended by the World Health Organization. Tumor size, local depth of invasion (DOI), margin status, and lymph node metastasis were determined on pathologic examination. The final disease stage was determined by a combination of surgical and pathologic findings according to the current tumor-node-metastasis staging system for oral cancer. Follow-up data were obtained from the patients' medical charts and our tumor registry service.

\subsection{0. mRNA Microarray Assay}

Total RNA was isolated from cell lines with Trizol (Invitrogen Corporation, Carlsbad, CA, USA). The Human OneArray v5 (Phalanx Biotech Group, Hsinchu, Taiwan) contains 30,275 DNA oligonucleotide probes, and each probe is a 60 mer probe designed in the sense direction. Among the probes, 29,187 probes corresponded to the annotated genes in the Refseq v38 (National Center for Biotechnology Information, Bethesda, MD, USA) and Ensembl v56 (Ensembl, Hinxton, Cambridge, UK) databases.

\subsection{Statistical Analysis}

Data are represented as mean \pm SEM. Statistical analyses were performed using an unpaired, two-tailed Student's $t$ test, and the values are expressed as means with $95 \%$ confidence intervals. A $p$-value of $<0.05$ was considered statistically significant. The correlation between the clinicopathological parameters of patients with oral cancer and the expression of LIF was analyzed using the Kruskal-Wallis test. The correlation between LIF expression and habits related to betel nut chewing and tobacco smoking was analyzed using the chi-squared test. The associated prognostic factors were identified by the univariate and multivariate survival analyses with Cox proportional hazards regression model using SAS 9.1 (SAS Institute Inc., Morrisville, NC, USA). 


\section{Results}

\subsection{LIF Affects Regional Lymph Node Involvement and the Advanced Stage of OSCC}

To study the clinical relevance of LIF in patients with OSCC, we first examined LIF protein expression in paraffin-embedded tumor tissue specimens of patients with OSCC through immunohistochemical analysis. Cytoplasmic LIF staining was found in nearly all cancer cells of the tumor nests for positive LIF staining (Figure 1). The grade of LIF expression in each specimen was further classified based on the percentage of tumor cells stained as follows: level $0(0 \%$ or $<5 \%)$, level 1 (5-25\%), level 2 (26-50\%), level 3 (51-75\%), and level 4 (>75\%). Of the 100 OSCC samples analyzed, negative (level 0), low (levels 1 and 2), and high (levels 3 and 4) expression levels of LIF were observed in $32 \%$ (32/100), 22\% (22/100), and 46\% (46/100) of OSCC specimens, respectively. The relationships between LIF expression in the initial biopsy specimens and clinical parameters of 100 patients with OSCC are shown in Table 1. Cancer with positive lymph node metastasis was associated with higher LIF expression $(p=0.022)$. Similarly, a significant association between LIF staining and advanced cancer staging (stages III and IV) ( $p=0.002)$ was noted. Generally, the larger the tumor size, the higher the LIF expression; however, no significant association was observed between LIF expression and tumor size $(p=0.051)$. We also found a significant association of LIF protein expression and other clinicopathological variables such as depth of invasion $(p=0.001)$ and surgical margins $(p=0.023)$. Furthermore, univariate analysis was used to investigate the relationships of LIF expression and cancer characteristics with patients' overall survival (Figure 2). Kaplan-Meier curves showed that OSCC patients with higher LIF expression, advanced stage, large tumor size, or positive lymph node metastasis had significantly shorter overall survival $(p<0.001, p=0.011, p=0.002$, and $p=0.014$, respectively; log-rank test) than others. Univariate and multivariate survival analyses were performed using a Cox proportion hazards regression model. Advanced lymph node metastasis $(p=0.041)$, poor histological differentiation $(p=0.027)$, a DOI of $5-9 \mathrm{~mm}(p=0.007)$, a DOI of $<5 \mathrm{~mm}(p=0.001)$, and advanced clinical stage $(p=0.001)$ were correlated with poor survival in the univariate analysis. Advanced clinical stage $(p=0.026)$ was identified as an independent unfavorable prognosis factor in the multivariate analysis (Table 2). In addition, the association between LIF and habits was evaluated. The details of patients' oral health habits, including the daily or weekly consumption of areca quid (AQ), cigarettes, and alcohol, as well as the duration of these habits, were recorded. Patients with OSCC were defined as AQ chewers when they chewed two or more AQs daily for at least 1 year. They were categorized as cigarette smokers when they smoked every day for at least 1 year and consumed more than 50 packs of cigarettes per year. Finally, they were classified as alcohol drinkers when they drank more than 4 days a week and consumed more than $20 \mathrm{~g}$ of pure alcohol per week for at least 1 year. According to these definitions, 79 (79\%) were drinkers, 80 (80\%) were AQ chewers, and 83 $(83 \%)$ were smokers. Finally, no significant correlation was found between LIF expression and areca nut chewing, tobacco smoking, and alcohol drinking (Table 3). This finding suggests that LIF plays an important role in the cancer progression of patients with OSCC.

\subsection{LIF Enhances Cell Migration and Invasion Abilities through Outside-In Signaling in OSCC Cells}

To clarify the correlation between LIF expression and cancer metastasis in oral cancer, we used OSCC cell lines to investigate whether LIF plays a critical role in OSCC progression. We first knocked down LIF expression by shRNA. Transiently transfected shLIF expression plasmid in SAS cells indicated that migrated and invaded cells were decreased compared with control cells (Figure 3A). We also knocked down LIF expression in Cal27 cell lines and confirmed that the cell motility was decreased in another cell line (Figure S1). By contrast, the invasion and migration capabilities were enhanced in LIF overexpressing CA9-22 cells compared with control cells (Figure 3B). 
A

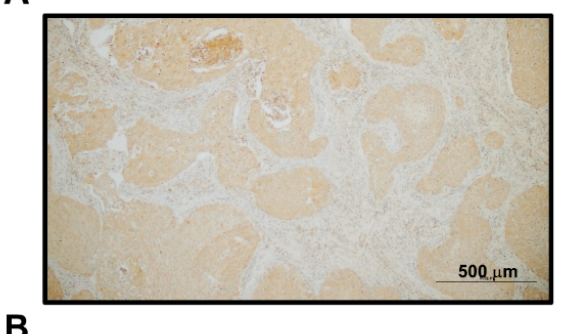

B

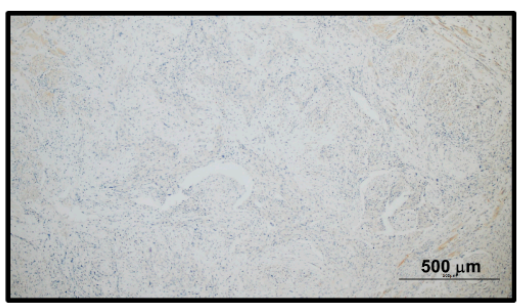

Figure 1. Immunohistochemical staining of LIF expression in patients with OSCC. LIF expression was determined by immunohistochemistry in paraffin-embedded tumor tissues of patients with OSCC. (A) Representative image of positive LIF staining in a patient with OSCC. The cytoplasmic LIF staining was found in nearly all cancer cells of the tumor nests. (B) Representative image of no LIF staining in a patient with OSCC.

A

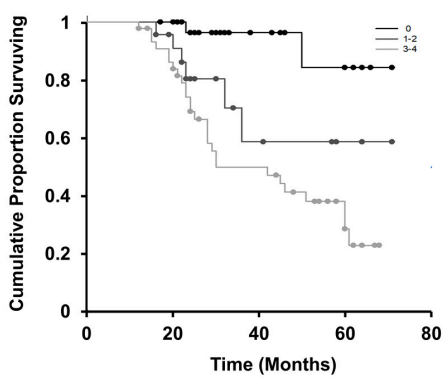

C

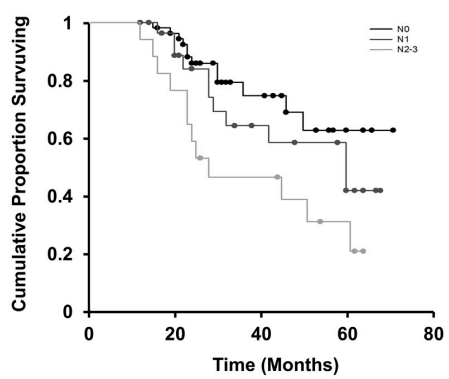

B

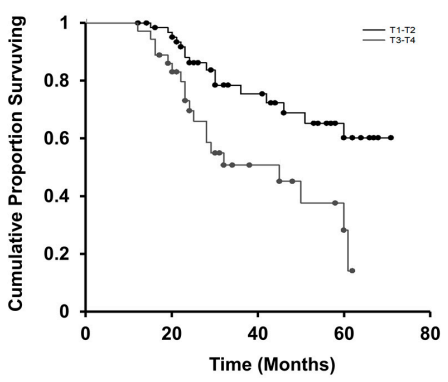

D

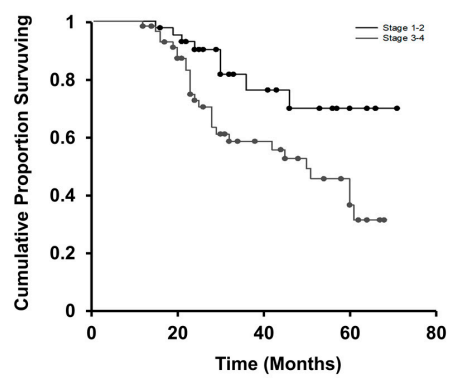

Figure 2. Kaplan-Meier survival curves of 100 patients with OSCC. (A) The cumulative survival for patients with none or a low degree (0-2) of LIF staining was significantly higher than that for patients with a high degree (3-4). (B) Overall survival was significantly lower in patients with a larger tumor size $(\mathrm{T} 3+\mathrm{T} 4)$ than in those with smaller tumor size $(\mathrm{T} 1+\mathrm{T} 2)(p=0.002)$. (C) Overall survival was significantly higher in patients without lymph node metastasis (N0) than in those with an advanced status of lymph node metastasis $(\mathrm{N} 2+\mathrm{N} 3)(p=0.014)$. (D) Overall survival was significantly shorter in patients with advanced-stage (stages 3-4) tumors than in those with earlier-stage (stages 1-2) tumors $(p=0.011)$. The duration of survival was measured from the beginning of treatment to the time of death (complete) or the last follow-up (censored). 
Table 1. Correlation between leukemia inhibitory factor (LIF) expression and clinicopathological parameters.

\begin{tabular}{|c|c|c|c|c|}
\hline \multirow{2}{*}{ Clinicopathological Parameters } & \multicolumn{4}{|c|}{ Degree of LIF Staining } \\
\hline & $0(n=32)$ & 1 or $2(n=22)$ & 3 or $4(n=46)$ & $p$-Value ${ }^{\text {a }}$ \\
\hline \multicolumn{5}{|l|}{ Patient's ages (years) } \\
\hline$<50$ & $7(21.9 \%)$ & $6(27.3 \%)$ & $11(23.9 \%)$ & 0.828 \\
\hline $50-59$ & $18(56.2 \%)$ & $9(40.9 \%)$ & $19(41.3 \%)$ & \\
\hline $60-69$ & $4(12.5 \%)$ & $5(22.7 \%)$ & $9(19.6 \%)$ & \\
\hline$\geq 70$ & $3(9.4 \%)$ & $2(9.1 \%)$ & $7(15.2 \%)$ & \\
\hline \multicolumn{5}{|l|}{ Patient's sex } \\
\hline Male & $28(87.5 \%)$ & $19(95.5 \%)$ & $44(95.6 \%)$ & 0.335 \\
\hline Female & $4(12.5 \%)$ & $3(4.5 \%)$ & $2(4.4 \%)$ & \\
\hline \multicolumn{5}{|l|}{ Cancer locations } \\
\hline Buccal and lip SCC & $16(50 \%)$ & $10(45.5 \%)$ & $22(47.8 \%)$ & $0.526^{\mathrm{b}}$ \\
\hline Gingival SCC & $5(15.6 \%)$ & $5(22.7 \%)$ & $2(4.3 \%)$ & \\
\hline Mouth floor SCC & $0(0 \%)$ & $0(0 \%)$ & $1(2.2 \%)$ & \\
\hline Palate SCC & $1(3.1 \%)$ & $1(4.5 \%)$ & $3(6.5 \%)$ & \\
\hline Tongue SCC & $10(31.3 \%)$ & $6(27.3 \%)$ & $18(39.1 \%)$ & \\
\hline \multicolumn{5}{|l|}{ T status } \\
\hline $\mathrm{T} 1-\mathrm{T} 2$ & $22(68.8 \%)$ & $19(86.4 \%)$ & $23(50 \%)$ & 0.051 \\
\hline T3-T4 & $10(31.2 \%)$ & $3(13.6 \%)$ & $23(50 \%)$ & \\
\hline \multicolumn{5}{|l|}{ N status } \\
\hline N0 & $23(71.9 \%)$ & $12(54.5 \%)$ & $18(39.1 \%)$ & $0.022 *$ \\
\hline N1 & $6(18.8 \%)$ & $4(18.2 \%)$ & $20(43.5 \%)$ & \\
\hline N2-N3 & $3(9.3 \%)$ & $6(27.3 \%)$ & $8(17.4 \%)$ & \\
\hline \multicolumn{5}{|l|}{ Clinical staging } \\
\hline Stage 1 & $10(31.25 \%)$ & $5(22.7 \%)$ & $5(10.9 \%)$ & $0.022 *$ \\
\hline Stage 2 & $10(31.25 \%)$ & $7(31.8 \%)$ & $6(13.0 \%)$ & \\
\hline Stage 3 & $2(6.25 \%)$ & $3(13.6 \%)$ & $13(28.3 \%)$ & \\
\hline Stage 4 & $10(31.25 \%)$ & $7(31.8 \%)$ & $22(47.8 \%)$ & \\
\hline Stages 1-2 & $20(62.5 \%)$ & $12(54.5 \%)$ & $11(23.9 \%)$ & $0.002 *$ \\
\hline Stages 3-4 & $12(37.5 \%)$ & $10(45.5 \%)$ & $35(76.1 \%)$ & \\
\hline \multicolumn{5}{|l|}{ Histological differentiation } \\
\hline Well-diff. SCC & $30(93.8 \%)$ & $20(90.1 \%)$ & $41(89.1 \%)$ & 0.773 \\
\hline Moderately-diff. SCC & $1(3.1 \%)$ & $2(9.9 \%)$ & $3(6.5 \%)$ & \\
\hline Poorly-diff. SCC & $1(3.1 \%)$ & $0(0.0 \%)$ & $2(4.4 \%)$ & \\
\hline \multicolumn{5}{|l|}{ Depth of invasion (DOI) } \\
\hline$<5 \mathrm{~mm}$ & $25(78.1 \%)$ & $13(59.1 \%)$ & $9(19.6 \%)$ & $0.001 *$ \\
\hline $5-9 \mathrm{~mm}$ & $5(15.6 \%)$ & $5(22.7 \%)$ & $29(63.0 \%)$ & \\
\hline$>9 \mathrm{~mm}$ & $2(6.3 \%)$ & $4(18.2 \%)$ & $8(17.4 \%)$ & \\
\hline \multicolumn{5}{|l|}{ Margin status } \\
\hline$\geq 5 \mathrm{~mm}$ & $29(90.6 \%)$ & $17(72.3 \%)$ & $27(58.7 \%)$ & $0.023 *$ \\
\hline$<5 \mathrm{~mm}$ & $1(3.1 \%)$ & $4(18.2 \%)$ & $15(32.6 \%)$ & \\
\hline Involved & $2(6.3 \%)$ & $1(4.5 \%)$ & $4(8.7 \%)$ & \\
\hline \multicolumn{5}{|l|}{ Perineural invasion } \\
\hline No & $27(84.4 \%)$ & $18(81.8 \%)$ & $36(78.3 \%)$ & 0.79 \\
\hline Yes & $5(15.6 \%)$ & $4(18.2 \%)$ & $10(21.7 \%)$ & \\
\hline
\end{tabular}

\footnotetext{
${ }^{\mathrm{a}}$ Kruskal-Wallis test, ${ }^{\mathrm{b}}$ Based on a chi-squared test. Abbreviation: SCC—-squamous cell carcinoma. $\left({ }^{*} p<0.05\right)$.
} 
Table 2. Univariate and multivariate survival analyses of LIF and clinicopathological parameters in patients with oral squamous cell carcinoma (OSCC).

\begin{tabular}{lcc}
\hline \multicolumn{1}{c}{ Factor } & Hazard Ratio (95\% CI) & $p$-Value ${ }^{\text {a }}$ \\
\hline Univariate & & 0.062 \\
Cancer locations (palatal vs. buccal and lip) & $4.95(0.92-26.88)$ & 0.22 \\
Cancer locations (palatal vs. gingival) & $3.52(0.46-26.73)$ & 0.172 \\
Cancer locations (palatal vs. tongue) & $4.18(0.53-32.68)$ & 0.217 \\
T status (T3 + T4 vs. T1 + T2) & $1.44(0.30-6.94)$ & 0.314 \\
N status (N2 + N3 vs. N0) & $2.78(0.37-20.45)$ & $0.041^{*}$ \\
N status (N2 + N3 vs. N1) & $2.91(1.04-8.06)$ & 0.481 \\
Clinical staging (stages 3 + 4 vs. 1 + 2) & $0.48(0.06-3.64)$ & $0.027^{*}$ \\
Histological differentiation (poor vs. well) & $13.10(1.35-127.41)$ & 0.81 \\
Histological differentiation (moderate vs. well) & $1.17(0.31-4.33)$ & $0.007^{*}$ \\
DOI (>10 mm vs. 5-9 mm) & $5.43(1.58-18.66)$ & $0.001^{*}$ \\
DOI (>10 mm vs. <5 mm) & $16.08(2.96-87.71)$ & 0.435 \\
Margin status (margin involved vs. A > 5 mm) & $1.88(0.38-9.26)$ & 0.104 \\
Margin status (margin involved vs. closed $<5$ mm) & $4.95(0.71-34.48)$ & 0.817 \\
PNI (negative vs. positive) & $0.87(0.28-2.72)$ & $0.001^{*}$ \\
LIF label index (3 + 4 vs. 0) & $8.84(5.71-136.99)$ & 0.05 \\
LIF label index (3 + 4 vs. 1 + 2) & $1.36(0.0-2.7)$ & \\
\hline Multivariate & & $0.026^{*}$ \\
LIF Label index (3 + 4 vs. 0) & $6.83(2.88-133.68)$ & \\
\hline
\end{tabular}

${ }^{a}$ Based on a Cox regression—-proportion hazards model test. Abbreviation: CI-confidence interval. PNI: Perineural Invasion. $\left({ }^{*} p<0.05\right)$.

Table 3. Correlation between LIF expression and habits of betel nut chewing and tobacco smoking.

\begin{tabular}{|c|c|c|c|c|}
\hline \multirow{2}{*}{ Clinicopathological Parameters } & \multicolumn{4}{|c|}{ Degree of LIF Staining } \\
\hline & $0(n=32)$ & 1 or $2(n=22)$ & 3 or $4(n=46)$ & $p$-Value ${ }^{\text {a }}$ \\
\hline \multicolumn{5}{|l|}{ Daily alcohol consumption } \\
\hline Nondrinkers & $6(18.8 \%)$ & $5(22.7 \%)$ & $10(21.7 \%)$ & 0.997 \\
\hline$\leq 3500 \mathrm{~mL}$ & $17(53.1 \%)$ & $11(50 \%)$ & $23(50.00 \%)$ & \\
\hline$>3500 \mathrm{~mL}$ & $9(28.1 \%)$ & $6(27.3 \%)$ & $13(28.3 \%)$ & \\
\hline \multicolumn{5}{|l|}{ Duration of drinking alcohol } \\
\hline Nondrinkers & $6(18.8 \%)$ & $5(22.7 \%)$ & $10(21.7 \%)$ & 0.794 \\
\hline$\leq 10$ years & $7(21.9 \%)$ & $7(31.8 \%)$ & $9(19.6 \%)$ & \\
\hline$>10$ years & $19(59.3 \%)$ & $10(45.5 \%)$ & $27(58.7 \%)$ & \\
\hline \multicolumn{5}{|l|}{ Daily AQ consumption } \\
\hline Nonchewers & $10(31.3 \%)$ & $4(18.2 \%)$ & $5(10.9 \%)$ & 0.176 \\
\hline$\leq 10$ quids & $10(31.2 \%)$ & $7(31.8 \%)$ & $22(47.8 \%)$ & \\
\hline$>10$ quids & $12(37.5 \%)$ & $11(50 \%)$ & $19(41.3 \%)$ & \\
\hline \multicolumn{5}{|l|}{ Duration of chewing AQs } \\
\hline Nonchewers & $10(31.3 \%)$ & $4(18.2 \%)$ & $5(10.9 \%)$ & 0.219 \\
\hline$\leq 10$ years & $5(15.6 \%)$ & $6(27.3 \%)$ & $13(28.3 \%)$ & \\
\hline$>10$ years & $17(53.1 \%)$ & $12(54.5 \%)$ & $28(60.8 \%)$ & \\
\hline \multicolumn{5}{|l|}{ Daily cigarette consumption } \\
\hline Nonsmokers & $6(18.8 \%)$ & $3(13.6 \%)$ & $8(17.4 \%)$ & 0.487 \\
\hline$\leq 1$ pack & $17(53.1 \%)$ & $15(68.2 \%)$ & $21(45.6 \%)$ & \\
\hline$>1$ pack & $9(28.1 \%)$ & $4(18.2 \%)$ & $17(37.0 \%)$ & \\
\hline \multicolumn{5}{|l|}{ Duration of smoking } \\
\hline Nonsmokers & $6(18.8 \%)$ & $3(13.6 \%)$ & $8(17.4 \%)$ & 0.092 \\
\hline$\leq 10$ years & $2(6.2 \%)$ & $2(9.1 \%)$ & $4(8.7 \%)$ & \\
\hline$>10$ years & $24(75 \%)$ & $17(77.2 \%)$ & $34(73.9 \%)$ & \\
\hline
\end{tabular}

${ }^{a}$ Based on a chi-square test. Abbreviation: AQ-areca quid. 
A
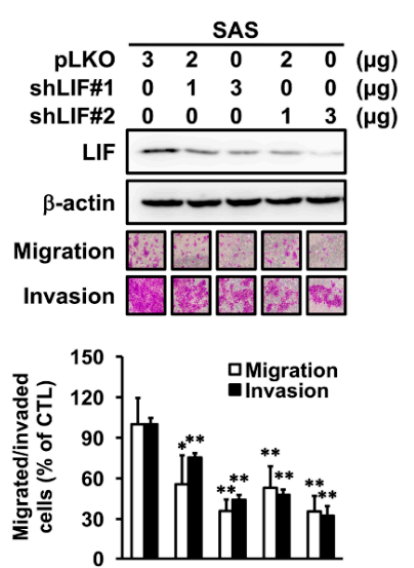

C

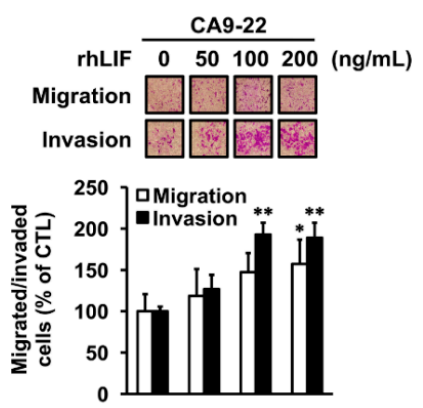

B
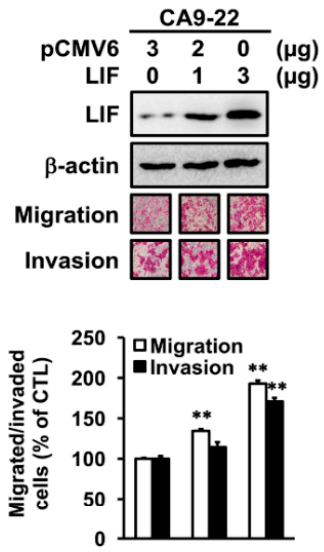

D

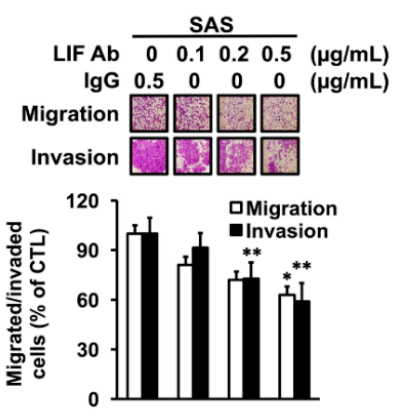

Figure 3. Invasion and migration ability of LIF in OSCC cells. (A,B) Western blot analysis of LIF expression in CA9-22 or SAS cells transiently transfected with LIF-expressed or shLIF plasmid. $\beta$-actin was used as an internal control. An in vitro migration and invasion assay was used to evaluate cell migration and invasion ability, performed for 24 or $48 \mathrm{~h}\left({ }^{*} p<0.05,{ }^{* *} p<0.01\right)$. (C,D) CA9-22 and SAS cells were subcultured in a Boyden chamber and treated with various concentrations of recombinant LIF protein (rLIF) or LIF neutralization antibody overnight. Cell motility toward the lower face of the filter was observed and quantified $(* p<0.05, * * p<0.01)$.

LIF can be secreted and detected in the cell matrix [26]. To confirm if LIF regulates cell motility through outside-in or inside-out signaling, recombinant LIF protein (rLIF) and LIF neutralization antibody were used. rLIF significantly increased the migration and invasion of CA9-22 cells in a dose-dependent manner (Figure 3C). In addition, LIF neutralization antibody decreased the motility of SAS cells in a dose-dependent manner (Figure 3D), indicating that LIF induced cell motility through outside-in signaling. With such results taken together, we concluded that LIF has direct roles in enhancing OSCC metastasis.

\subsection{INHBA is a Key Downstream Effector in LIF-Enhanced OSCC Progression}

As LIF induced tumor cell metastasis ability, we investigated the potential downstream effector(s) of LIF in OSCC progression; thus, a mRNA microarray was performed. According to Figure 4A, the heatmap of stable LIF transfectants showed 22 genes that were substantially regulated by LIF. To further analyze the function of LIF in our array data, gene set enrichment analysis (GSEA) was conducted, which showed that the metastasis-associated genes were significantly downregulated in LIF knockdown Cal27 cells compared with control clone (Figure 4B). We next validated the mRNA expression of metastasis-related genes in the microarray analysis, including ubiquitin-conjugating enzyme E2 C (UBE2C), INHBA, CD44 molecule (CD44), mitotic checkpoint serine/threonine kinase (BUB1), and baculoviral IAP repeat containing 5 (BIRC5) by quantitative real-time PCR (Figure $4 C$ ). The mRNA 
expression of INHBA was remarkably decreased in LIF knockdown Cal27 cells. As observed with microarray findings, the INHBA mRNA expression was upregulated by overexpressed LIF and downregulated by knockdown LIF expression in OSCC cell lines (Figure 4D). We thus hypothesized that INHBA might be a potential downstream target gene of LIF.

A

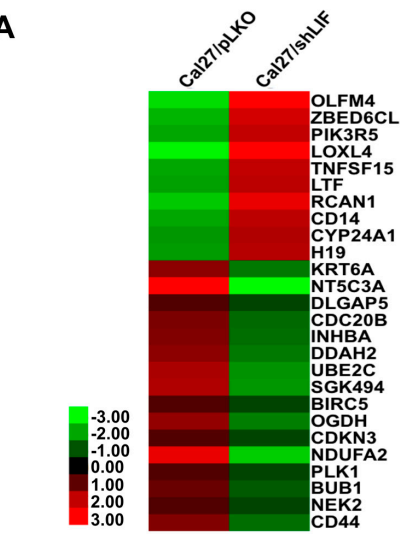

B

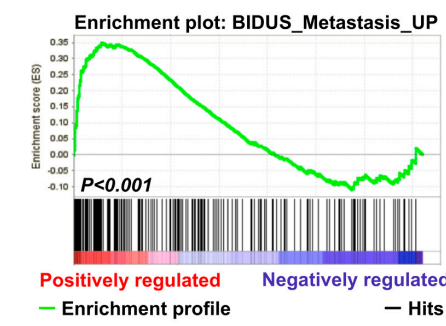

C

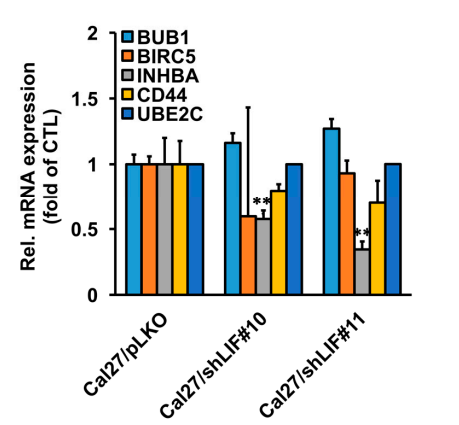

D

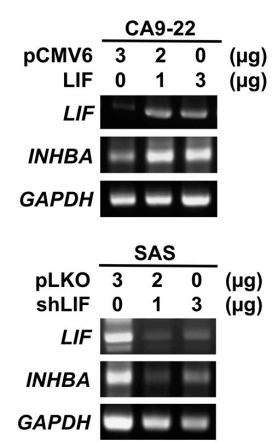

E

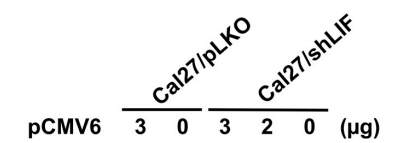

$\begin{array}{lllllll}\text { INHBA } & 0 & 3 & 0 & 1 & 3 & (\mu \mathrm{g})\end{array}$

INHBA

LIF

$\beta$-actin

Migration

Invasion

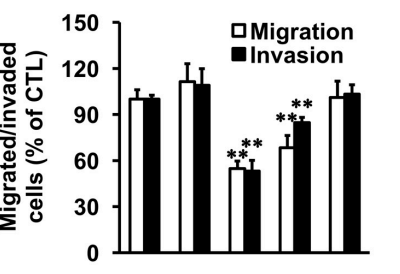

$\mathbf{F}$

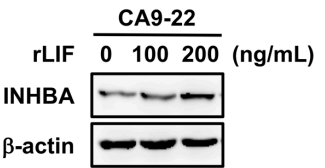

Figure 4. Inhibin beta A subunit (INHBA) as the major downstream effector in LIF increases oral cancer progression. (A) Heatmap of mRNA expression profile in Cal27/pLKO and Cal27/shLIF stable clones. (B) Gene set enrichment analysis (GSEA) showed the enrichment of metastatic genes in Cal27/pLKO versus Cal27/shLIF cells. (C) Real-time PCR analysis of BUB1, BIRC5, INHBA, CD44, and UBE2C mRNA expression in Cal27/pLKO and Cal27/shLIF cells $(* * p<0.01)$. (D) Reverse transcription PCR analysis of INHBA mRNA expression in CA9-22 and HSC3 cells transiently transfected LIF-expressed or shLIF plasmids. (E) Cal27/pLKO and Cal27/shLIF stable clones were seeded and transiently transfected with $3 \mu \mathrm{g}$ of control plasmid or various concentrations of INHBA plasmids and incubated for $48 \mathrm{~h}$, then subcultured in a Boyden chamber overnight. Cell motility toward the lower face of the filter was observed and quantified $\left({ }^{* *} p<0.01\right)$. (F) Western blot analysis of INHBA protein expression in CA9-22 cells after they were treated with rLIF. $\beta$-actin was used as an internal control.

To evaluate whether INHBA is a major downstream effector in LIF-induced OSCC metastasis, we transiently transfected IHNBA-expressing plasmids with LIF knockdown transfectants in a dose-dependent manner and then determined the cells' migration and invasion abilities. This transient transfection with INHBA plasmids in LIF knockdown transfectants substantially restored the 
shLIF-decreased INHBA protein expression and cell motility (Figure 4E). To investigate whether the INHBA protein expression was regulated by LIF through outside-in signaling, rLIF treatment of CA9-22 cells was performed, which considerably increased the INHBA protein expression (Figure 4F). These findings suggest that INHBA is a potential downstream effector in LIF-induced OSCC cancer progression.

\subsection{Relationship between LIF and INHBA Expression in Patients with OSCC}

To validate our findings, we determined the correlation between LIF and INHBA mRNA expression in patients with oral cancer by quantitative real-time PCR. As Figure 5 shows, INHBA mRNA expression was moderately correlated with LIF mRNA expression in patients with OSCC $\left(R^{2}=0.67\right)$. The result supports the assumption that, in vitro, INHBA influences LIF-associated metastasis in oral cancer.

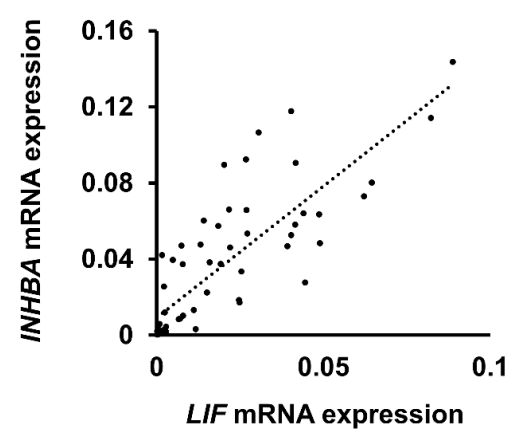

Figure 5. INHBA was moderately correlated with LIF in patients with OSCC. Correlation between LIF and INHBA in patients with OSCC $\left(R^{2}=0.67\right)$. The mRNA levels of patients' samples were measured using real-time PCR. Data are presented as mean \pm SD for mRNA expression.

\section{Discussion}

LIF has been well studied as a tumorigenesis promotor or suppressor in different types of tumors. OSCC is the most prevalent malignant tumor of the head and neck region. Despite improvements in treatment, the five-year survival rate of patients with oral cancer has still not improved significantly. A major challenge of current basic and clinical research is to identify a novel molecular marker that can improve the treatment of oral cancer.

In this study, we identified a relationship between LIF and major risk factors for oral cancer such as tobacco smoking, betel nut chewing, and alcohol consumption. The results demonstrated that no significant correlation existed between LIF and these habits. On the basis of our findings, LIF was not involved in the mechanisms of tobacco smoking, betel nut chewing, and alcohol consumption related to oral cancer. Whether LIF participates in carcinogenesis remains controversial. However, we found that LIF is essential for oral cancer transformation and malignant progression. LIF can promote cancer cell progression, including migration and invasion, through INHBA in OSCC cells. INHBA encoding inhibin $\beta$ A subunit dimerizes with another $\beta$ subunit to make activin. Activin enhances Follicle Stimulating Hormone (FSH) biosynthesis and secretion and participates in menstrual cycle regulation [27]. Studies have revealed that activin is overexpressed in OSCC compared with normal oral mucosa, and that high activin A levels are significantly associated with lymph node metastasis, tumor differentiation, and poor survival [28,29]. LIF overexpression enhanced INHBA expression to regulate cancer cell motility, whereas LIF knockdown reduced cell motility and INHBA expression in shLIF transfectants. This suggests that LIF and INHBA are essential for OSCC progression. We found that patients with positive lymph node metastasis had higher LIF expression. However, high LIF staining was also found in patients with advanced cancer stages. These findings suggest that LIF plays an important role in the cancer progression of patients with OSCC.

The role of LIF in multiple cancers is variable. It acts as an oncogene in rhabdomyosarcoma [24], nasopharyngeal carcinoma [18], and colorectal cancer [25], but it acts as a tumor suppressor in several 
cancer types, including breast cancer [22,23], melanoma [21], and hepatocellular carcinoma [30]. Research has indicated that some genes have both oncogenic and tumor-suppressing potential in various cancer types [31-34]. Cancer is a somatic mutation accumulation [35,36]. It has been mentioned that the gain-of-function mutations of these genes that hold dual roles might promote oncogenic functions, and the loss-of-function mutations downregulate the tumor-suppressing expression [31]. Additionally, the microenvironment around a tumor determines the direction of tumor development and metastasis [33]. The complex immune networks of immune cells and the cytokines surrounding the tumors influence the outcomes of anticancer activities or tumor promotion [33,37]. A gene can become a double-edged sword by either promoting or inhibiting tumor progression according to cancer types. Identifying the interaction between genes and cancers in the tumor microenvironment is crucial.

Ohata et al. demonstrated that OSCC stimulated cancer-associated fibroblasts (CAFs) to produce LIF, which induced cell invasion. However, the mechanism through which LIF promotes cancer motility remains unclear [38]. Based on our findings, rLIF and the transfection of LIF expression vector confirmed that both exogenous and endogenous LIF induced oral cancer motility. We identified INHBA as a potential downstream gene in LIF-associated OSCC progression using a high-throughput mRNA microarray. In a previous study, the overexpression of activin A in OSCC regulated cell apoptosis and invasiveness [28]. Activin A was also clinically correlated with lymph node metastasis and poor survival [28]. Therefore, we posit that CAF-produced or OSCC-produced LIF may be the upstream effector of INHBA in the control of OSCC metastasis (cell motility and invasion).

The results of the present study revealed that almost half of the patients with stage 4 cancer had low LIF expression (17/39). We observed that stage 4 patients with lower LIF expression had lower rates of regional lymph node metastasis and a longer survival time compared with stage 4 patients with high LIF expression. These findings also imply that patients who express low LIF tend to have a good prognosis. Although LIF expression does not exhibit a significant association with tumor status, higher LIF protein levels seem to be associated with more invasive cancer behavior, such as deeper invasion and regional lymph node metastasis. In clinical practice, if a patient presents with a small primary tumor with high LIF expression, the possibility of regional lymph node metastasis should be a concern. These findings suggest that LIF can be a valuable predictor in patients with local invasive cancer behavior, even in cases where the tumor status is not advanced.

\section{Conclusions}

In conclusion, LIF contributes to cancer progression by enhancing regional lymphatic spread, thereby leading to an advanced cancer stage. Regulation of LIF downstream molecules, such as INHBA, can thus inhibit cell invasion and migration. We demonstrated a novel mechanism (INHBA regulation) by which LIF influences cancer progression. LIF can thus be a potential diagnostic or therapeutic marker in patients with advanced OSCC stages.

Supplementary Materials: The following are available online at http://www.mdpi.com/2077-0383/9/2/295/s1, Figure S1: Knocked down LIF expression decreased the invasion and migration capabilities in Cal27 cell lines.

Author Contributions: Conceptualization, H.-H.C.; Methodology, H.-H.C.; Investigation; T.-A.L.; Writing-Original Draft Preparation, T.-A.L.; Data Curation, T.-A.L. and T.-S.W.; Validation, T.-S.W.; Formal Analysis, T.-A.L. and T.-S.W.; Writing-Review and Editing, Y.-J.L. and C.-N.Y.; Supervision, Y.-J.L.; Visualization, C.-N.Y.; Software, M.M.I.R.; Project Administration, M.M.I.R. All authors have read and agreed to the published version of the manuscript.

Funding: This work was supported by the National Science Council, Taiwan (NSC-105-2314-B-002-080).

Acknowledgments: This work was supported by the National Science Council of Taiwan (NSC-105-2314B-002-080). We sincerely thank Frank Obrock and Damien Watterson of Wallace Academic Editing for their assistance in critically reviewing and editing this manuscript. We extend a special thanks to Douglas Ellsworth for manuscript writing assistance.

Conflicts of Interest: The authors declare that they have no competing interests. 


\section{References}

1. Tian, S.; Jiang, C.; Liu, X.; Xu, S.; Zhang, Z.; Chen, H.; Zhang, Y.; Liu, Y.; Ma, D. Hypermethylation of IFN-gamma in oral cancer tissues. Clin. Oral Investig. 2017, 21, 2535-2542. [CrossRef]

2. Johnson, N.W.; Jayasekara, P.; Amarasinghe, A.A. Squamous cell carcinoma and precursor lesions of the oral cavity, epidemiology and aetiology. Periodontol. 2000 2011, 57, 19-37. [CrossRef]

3. Yang, X.; Ruan, H.; Hu, X.; Cao, A.; Song, L. miR-381-3p suppresses the proliferation of oral squamous cell carcinoma cells by directly targeting FGFR2. Am. J. Cancer Res. 2017, 7, 913-922.

4. Brocklehurst, P.; Kujan, O.; O'Malley, L.A.; Ogden, G.; Shepherd, S.; Glenny, A.M. Screening programmes for the early detection and prevention of oral cancer. Cochrane Database Syst. Rev. 2013, 11, CD004150. [CrossRef] [PubMed]

5. Hung, K.F.; Lai, K.C.; Liu, T.Y.; Liu, C.J.; Lee, T.C.; Lo, J.F. Asb6 upregulation by Areca nut extracts is associated with betel quid-induced oral carcinogenesis. Oral Oncol. 2009, 45, 543-548. [CrossRef] [PubMed]

6. Chen, C.L.; Chi, C.W.; Chang, K.W.; Liu, T.Y. Safrole-like DNA adducts in oral tissue from oral cancer patients with a betel quid chewing history. Carcinogenesis 1999, 20, 2331-2334. [CrossRef] [PubMed]

7. Sasahira, T.; Kirita, T.; Kuniyasu, H. Update of molecular pathobiology in oral cancer: A review. Int. J. Clin. Oncol. 2014, 19, 431-436. [CrossRef]

8. Li, W.C.; Lee, P.L.; Chou, I.C.; Chang, W.J.; Lin, S.C.; Chang, K.W. Molecular and cellular cues of diet-associated oral carcinogenesis-With an emphasis on areca-nut-induced oral cancer development. J. Oral Pathol. Med. 2015, 44, 167-177. [CrossRef]

9. Wang, T.Y.; Peng, C.Y.; Lee, S.S.; Chou, M.Y.; Yu, C.C.; Chang, Y.C. Acquisition cancer stemness, mesenchymal transdifferentiation, and chemoresistance properties by chronic exposure of oral epithelial cells to arecoline. Oncotarget 2016, 7, 84072-84081. [CrossRef]

10. Hsieh, C.H.; Hsu, H.H.; Shibu, M.A.; Day, C.H.; Bau, D.T.; Ho, C.C.; Lin, Y.M.; Chen, M.C.; Wang, S.H.; Huang, C.Y. Down-regulation of beta-catenin and the associated migration ability by Taiwanin $\mathrm{C}$ in arecoline and 4-NQO-induced oral cancer cells via GSK-3beta activation. Mol. Carcinog. 2017, 56, 1055-1067. [CrossRef]

11. Chang, M.C.; Chan, C.P.; Chen, Y.J.; Hsien, H.C.; Chang, Y.C.; Yeung, S.Y.; Jeng, P.Y.; Cheng, R.H.; Hahn, L.J.; Jeng, J.H. Areca nut components stimulate ADAM17, IL-1alpha, PGE2 and 8-isoprostane production in oral keratinocyte: Role of reactive oxygen species, EGF and JAK signaling. Oncotarget 2016, 7, 16879-16894. [PubMed]

12. Tsai, K.Y.; Su, C.C.; Chiang, C.T.; Tseng, Y.T.; Lian, I.B. Environmental heavy metal as a potential risk factor for the progression of oral potentially malignant disorders in central Taiwan. Cancer Epidemiol. 2017, 47, 118-124. [CrossRef] [PubMed]

13. Tsai, S.T.; Wong, T.Y.; Ou, C.Y.; Fang, S.Y.; Chen, K.C.; Hsiao, J.R.; Huang, C.C.; Lee, W.T.; Lo, H.I.; Huang, J.S.; et al. The interplay between alcohol consumption, oral hygiene, ALDH2 and ADH1B in the risk of head and neck cancer. Int. J. Cancer 2014, 135, 2424-2436. [CrossRef] [PubMed]

14. Cheng, E.H.; Liu, J.Y.; Lee, T.H.; Huang, C.C.; Chen, C.I.; Huang, L.S.; Lee, M.S. Requirement of Leukemia Inhibitory Factor or Epidermal Growth Factor for Pre-Implantation Embryogenesis via JAK/STAT3 Signaling Pathways. PLoS ONE 2016, 11, e0153086. [CrossRef]

15. Smith, A.G.; Nichols, J.; Robertson, M.; Rathjen, P.D. Differentiation inhibiting activity (DIA/LIF) and mouse development. Dev. Biol. 1992, 151, 339-351. [CrossRef]

16. Gearing, D.P.; Gough, N.M.; King, J.A.; Hilton, D.J.; Nicola, N.A.; Simpson, R.J.; Nice, E.C.; Kelso, A.; Metcalf, D. Molecular cloning and expression of cDNA encoding a murine myeloid leukaemia inhibitory factor (LIF). EMBO J. 1987, 6, 3995-4002. [CrossRef]

17. Junk, D.J.; Bryson, B.L.; Jackson, M.W. HiJAK'd Signaling; the STAT3 Paradox in Senescence and Cancer Progression. Cancers 2014, 6, 741-755. [CrossRef]

18. Liu, S.C.; Tsang, N.M.; Chiang, W.C.; Chang, K.P.; Hsueh, C.; Liang, Y.; Juang, J.L.; Chow, K.P.; Chang, Y.S. Leukemia inhibitory factor promotes nasopharyngeal carcinoma progression and radioresistance. J. Clin. Investig. 2013, 123, 5269-5283. [CrossRef]

19. Morton, S.D.; Cadamuro, M.; Brivio, S.; Vismara, M.; Stecca, T.; Massani, M.; Bassi, N.; Furlanetto, A.; Joplin, R.E.; Floreani, A.; et al. Leukemia inhibitory factor protects cholangiocarcinoma cells from drug-induced apoptosis via a PI3K/AKT-dependent Mcl-1 activation. Oncotarget 2015, 6, 26052-26064. [CrossRef] 
20. Yue, X.; Zhao, Y.; Zhang, C.; Li, J.; Liu, Z.; Liu, J.; Hu, W. Leukemia inhibitory factor promotes EMT through STAT3-dependent miR-21 induction. Oncotarget 2016, 7, 3777-3790. [CrossRef]

21. Humbert, L.; Ghozlan, M.; Canaff, L.; Tian, J.; Lebrun, J.J. The leukemia inhibitory factor (LIF) and p21 mediate the TGFbeta tumor suppressive effects in human cutaneous melanoma. BMC Cancer 2015, 15, 200. [CrossRef] [PubMed]

22. Hergovich, A. YAP-Hippo signalling downstream of leukemia inhibitory factor receptor: Implications for breast cancer. Breast Cancer Res. 2012, 14, 326. [CrossRef] [PubMed]

23. Chen, D.; Sun, Y.; Wei, Y.; Zhang, P.; Rezaeian, A.H.; Teruya-Feldstein, J.; Gupta, S.; Liang, H.; Lin, H.K.; Hung, M.C.; et al. LIFR is a breast cancer metastasis suppressor upstream of the Hippo-YAP pathway and a prognostic marker. Nat. Med. 2012, 18, 1511-1517. [CrossRef] [PubMed]

24. Wysoczynski, M.; Miekus, K.; Jankowski, K.; Wanzeck, J.; Bertolone, S.; Janowska-Wieczorek, A.; Ratajczak, J.; Ratajczak, M.Z. Leukemia inhibitory factor: A newly identified metastatic factor in rhabdomyosarcomas. Cancer Res. 2007, 67, 2131-2140. [CrossRef] [PubMed]

25. Yu, H.; Yue, X.; Zhao, Y.; Li, X.; Wu, L.; Zhang, C.; Liu, Z.; Lin, K.; Xu-Monette, Z.Y.; Young, K.H.; et al. LIF negatively regulates tumour-suppressor p53 through Stat3/ID1/MDM2 in colorectal cancers. Nat. Commun. 2014, 5, 5218. [CrossRef]

26. Li, X.; Yang, Q.; Yu, H.; Wu, L.; Zhao, Y.; Zhang, C.; Yue, X.; Liu, Z.; Wu, H.; Haffty, B.G.; et al. LIF promotes tumorigenesis and metastasis of breast cancer through the AKT-mTOR pathway. Oncotarget 2014, 5, 788-801. [CrossRef]

27. Vale, W.; Rivier, J.; Vaughan, J.; McClintock, R.; Corrigan, A.; Woo, W.; Karr, D.; Spiess, J. Purification and characterization of an FSH releasing protein from porcine ovarian follicular fluid. Nature 1986, 321, 776-779. [CrossRef]

28. Bufalino, A.; Cervigne, N.K.; de Oliveira, C.E.; Fonseca, F.P.; Rodrigues, P.C.; Macedo, C.C.; Sobral, L.M.; Miguel, M.C.; Lopes, M.A.; Paes Leme, A.F.; et al. Low miR-143/miR-145 Cluster Levels Induce Activin A Overexpression in Oral Squamous Cell Carcinomas, Which Contributes to Poor Prognosis. PLoS ONE 2015, 10, e0136599. [CrossRef]

29. Lee, H.Y.; Li, C.C.; Huang, C.N.; Li, W.M.; Yeh, H.C.; Ke, H.L.; Yang, K.F.; Liang, P.I.; Li, C.F.; Wu, W.J. INHBA overexpression indicates poor prognosis in urothelial carcinoma of urinary bladder and upper tract. J. Surg. Oncol. 2015, 111, 414-422. [CrossRef]

30. Luo, Q.; Wang, C.; Jin, G.; Gu, D.; Wang, N.; Song, J.; Jin, H.; Hu, F.; Zhang, Y.; Ge, T.; et al. LIFR functions as a metastasis suppressor in hepatocellular carcinoma by negatively regulating phosphoinositide 3-kinase/AKT pathway. Carcinogenesis 2015, 36, 1201-1212. [CrossRef]

31. Shen, L.; Shi, Q.; Wang, W. Double agents: Genes with both oncogenic and tumor-suppressor functions. Oncogenesis 2018, 7, 25. [CrossRef] [PubMed]

32. Yang, L.; Han, Y.; Suarez Saiz, F.; Minden, M.D. A tumor suppressor and oncogene: The WT1 story. Leukemia 2007, 21, 868-876. [CrossRef] [PubMed]

33. Zamarron, B.F.; Chen, W. Dual roles of immune cells and their factors in cancer development and progression. Int. J. Biol. Sci. 2011, 7, 651-658. [CrossRef] [PubMed]

34. Carafa, V.; Altucci, L.; Nebbioso, A. Dual Tumor Suppressor and Tumor Promoter Action of Sirtuins in Determining Malignant Phenotype. Front. Pharmacol. 2019, 10, 38. [CrossRef] [PubMed]

35. Wu, S.; Powers, S.; Zhu, W.; Hannun, Y.A. Substantial contribution of extrinsic risk factors to cancer development. Nature 2016, 529, 43-47. [CrossRef] [PubMed]

36. Reya, T.; Morrison, S.J.; Clarke, M.F.; Weissman, I.L. Stem cells, cancer, and cancer stem cells. Nature 2001, 414, 105-111. [CrossRef] [PubMed]

37. Shrihari, T.G. Dual role of inflammatory mediators in cancer. Ecancermedicalscience 2017, 11, 721. [CrossRef]

38. Ohata, Y.; Tsuchiya, M.; Hirai, H.; Yamaguchi, S.; Akashi, T.; Sakamoto, K.; Yamaguchi, A.; Ikeda, T.; Kayamori, K. Leukemia inhibitory factor produced by fibroblasts within tumor stroma participates in invasion of oral squamous cell carcinoma. PLoS ONE 2018, 13, e0191865. [CrossRef]

(C) 2020 by the authors. Licensee MDPI, Basel, Switzerland. This article is an open access article distributed under the terms and conditions of the Creative Commons Attribution (CC BY) license (http://creativecommons.org/licenses/by/4.0/). 